\title{
Enhanced healing and anti-inflammatory effects of a carbohydrate polymer with zinc oxide in patients with chronic venous leg ulcers: preliminary results
}

\author{
Mario Adan Moreno-Eutimio ${ }^{1}$, Lorena Espinosa-Monroy², Tania Orozco-Amaro ${ }^{1}$, \\ Yessica Torres-Ramos ${ }^{3}$, Araceli Montoya-Estrada ${ }^{3}$, Juan Jose Hicks ${ }^{4}$, Ernesto Rodríguez-Ayala ${ }^{5}$, \\ Pamela Del Moral', Jose Moreno ${ }^{1}$, Jorge Cueto-García ${ }^{5}$
}

\author{
${ }^{1}$ Immunobiology Laboratory, Hospital Juarez de México, Ministry of Health, Mexico \\ City, Mexico \\ ${ }^{2}$ Wound and Ostomy Clinic, Division of Research, Hospital Juarez de México, Ministry \\ of Health, Mexico City, Mexico \\ ${ }^{3}$ Biochemistry Department, National Institute of Perinatology, Ministry of Health, \\ Mexico City, Mexico \\ ${ }^{4}$ Health Research Policies, Coordinating Commission of National Health Institutes and \\ Highly Specialized Hospitals, Mexico City, Mexico \\ ${ }^{5}$ Health Sciences Faculty, Anahuac University, State of Mexico, Mexico
}

Submitted: 23 January 2015

Accepted: 1 August 2015

Arch Med Sci 2018; 14, 2: 336-344

DOI: 10.5114 /aoms.2016.59851

Copyright (c) 2016 Termedia \& Banach

\section{Abstract}

Introduction: Insufficient wound healing related to chronic inflammation of chronic venous leg ulcers (CVUs) represents an important public health problem. The aim of this study was to evaluate the effects of a carbohydrate polymer with zinc oxide therapy on CVUs.

Material and methods: Forty patients with CVUs were recruited for this study and were divided into a study group and control group. Patients In the study group were instructed to use venous compression treatment andtopical carbohydrate polymer with zinc oxide twice daily, while patients In the control group were treated with only venous compression treatment. All patients were followed up for 8 weeks. Peripheral blood samples and biosy tissue specimens were obtained at the initiation of treatment and after 8 weeks to assess serum levels of inflammatory cytokines as well as the percentage of leukocytes, T-helper cells, cytotoxic-T cells, macrophages and endothelial cells in the biopsy tissue using flow cytometry.

Results: A significantly greater reduction in the mean percentage ulcer area from baseline to eight weeks was observed in the study group (up to $40 \%$ for large ulcers). Furthermore, the patients in the study group had reduced systemic levels of the pro-inflammatory cytokines IL-8 $(p=0.0028)$ and IL-6 $(p=0.0302)$, fewer total CD45+ cells $(p=0.0038)$ and more CD $31+$ cells $(p=0.045)$ present in ulcer biopsies compared to the control group.

Conclusions: The carbohydrate polymer with zinc oxide treatment with venous compression enhances healing of CVUs and improves quality of life due, in part, to its anti-inflammatory properties.

Key words: chronic venous leg ulcers, carbohydrate polymer with zinc oxide, inflammation.

\author{
Corresponding author: \\ Mario Adan Moreno-Eutimio \\ $\mathrm{PhD}$ \\ Immunobiology Laboratory \\ Hospital Juarez de México \\ Ministry of Health \\ 5160 Instituto Politécnico \\ Nacional Avenue \\ 07760 Mexico City, Mexico \\ Phone: 015527419522 \\ E-mail: marioadan@ \\ inmunoquimica.com
}




\section{Introduction}

Chronic wounds are those that fail to heal by primary intention due to a persistent inflammatory phase of the normal healing process [1]. One of the most common types of chronic wounds is a chronic venous leg ulcer (CVU), which is thought to occur in $2-3 \%$ of the population. The high prevalence of CVUs greatly impacts public health programs due to high costs and prolonged treatments and incapacities [2]. Chronic venous leg ulcer $\mathrm{s}$ are associated with poor quality of life and loss of productivity due to pain, impaired mobility, social isolation, and depression [3].

Several therapeutic approaches have been proposed, but venous compressive therapy remains the gold standard treatment $[4,5]$. A recent systematic review assessed whether the use of advanced wound dressings, systemic antibiotics, or venous surgery enhanced the healing of venous ulcers compared to the use of adequate venous compression. This review concluded that most of the studies on advanced wound dressings that regulated moisture, facilitated debridement, included antimicrobial activity, or incorporated putative wound-healing accelerants did not demonstrate a statistically higher percentage of healed wounds compared with adequate compression with simple dressings [6].

Wound healing is a highly regulated process that involves interactions between extracellular matrix molecules, cytokines, resident cells, and infiltrating cell subtypes [7]. The immediate goal in the healing process is to achieve homeostasis and tissue integrity. Although inflammation recruits cells to the damaged tissue area for healing and remodeling, persistent inflammatory stimuli lead to inadequate healing in chronic wounds such as CVUs [8, 9].

Chronic inflammation in venous ulcers is not well understood, but several findings have shown that the lack of valvular function produces increased intravenous pressure and dilatation of endothelial pores that lead to fibrin deposition, edema and hypoxia. The inflammatory trap theory explains that several growth factors are trapped in the fibrin cuff, worsening the ischemia and hypoxia that contribute to the chronic inflammatory state [2, 10, 11]. Several studies have identified increased levels of pro-inflammatory cytokines, such as tumor necrosis factor $\alpha$ (TNF- $\alpha$ ), IL-8, and $\mathrm{IL}-1$, and growth factors that lead to many alterations in the normal healing process [9]. High expression of these inflammatory mediators is associated with the impairment of wound healing, suggesting that targeting cytokines may improve tissue repair [12-14].

Additionally, leukocyte infiltration around CVUs acts as a potential factor that increases and per- petuates chronic inflammation. Although these factors have not been completely elucidated, some studies show that inflammatory cells are crucial players during the healing process and that the alterations present in CVUs ultimately produce inadequate, delayed healing [15].

Our group has previously demonstrated that a carbohydrate polymer with zinc oxide has a potent anti-inflammatory effect on human mononuclear cells stimulated with lipopolysaccharide in vitro and that it also induced lower cell infiltration in an in vivo murine model of peritonitis [16]. This carbohydrate polymer with zinc oxide is a biodegradable, non-toxic adhesive based on a natural polymer that exerts anti-inflammatory activity similar to other naturally occurring polymers $[17,18]$.

Additionally, the zinc ion, a critical component of the carbohydrate polymer formulation, is an essential trace element that plays a crucial role as a cofactor for a number of enzymes. In addition, zinc exhibits different cellular activities and has been accepted and approved as a pharmaceutically acceptable additive that is widely used in dermatology as a wound-healing enhancer and in re-epithelialization of the skin. Furthermore, zinc ions have mild antibacterial and anti-inflammatory properties [17, 19, 20].

The purpose of this study was to assess the efficacy of a carbohydrate polymer with zinc oxide in accelerating the healing of CVUs by modifying the inflammatory state when it is incorporated into conventional venous compression treatment.

\section{Material and methods}

The carbohydrate polymer with zinc oxide (Pebisut) is a naturally occurring, complex carbohydrate polymer with a metal oxide that includes a zinc ion. Zinc plays an important role in healing processes. It has been approved by the U.S. Food and Drug Administration (FDA) as a pharmaceutically acceptable additive and is widely used in orthodontics and dermatology. The carbohydrate polymer with zinc oxide is biodegradable, nontoxic, does not require special storage conditions, does not allow microbial colonization, and has a shelf life of more than 1 year.

\section{Ethics statement}

The Hospital Juarez of Mexico Scientific Research Committee (composed of Scientific, Ethics and Bio-security Committees) approved the project (project number: HJM 2210/12-A), and the protocols that were used conformed to the ethical guidelines of the 1975 Declaration of Helsinki. The research protocol was approved by the Mexican Ministry of Health through the Federal Commission for Protection Against Sanitary Risks (COFEPRIS; 
registration no. 1853C2014SSA). All enrolled patients signed a written informed consent form.

\section{Patients}

Patients eligible to participate in the study had to be older than 18 years and had to have a confirmed diagnosis of CVU. Patients with ulcers of mixed or other etiology (diabetes, atherosclerosis, arterial hypertension, auto-immunologic diseases) were excluded. Patients were also excluded if they had a serious chronic active disease such as cancer or AIDS. Patients who were eligible to participate in the study were randomized to the venous compression treatment alone (control group, $n=20$ ) or a carbohydrate polymer with zinc oxide plus venous compression treatment (study group, $n=20$ ) groups using computer-generated random numbers. All groups received standard wound care once per week in the Wound and Ostomy Clinic of Hospital Juarez of Mexico for 8 weeks or until complete healing was achieved. In addition, therapy with a carbohydrate polymer with zinc oxide was applied to wounds twice daily for 8 weeks or until healing was complete. Standard treatment for CVUs included venous compression (Smith and Nephew, Hull, UK), non-adherent dressings and cleansing. The study participants included 40 outpatients (mean age: 61.15 years, $42.5 \%$ of patients male, mean body mass index: $27.8 \mathrm{~kg} / \mathrm{m}^{2}$, and mean baseline ulcer surface area 18.89 $\pm 14.38 \mathrm{~cm}^{2}$ ) with CVU. No significant difference was observed between the two groups with respect to age, sex or initial ulcer area.

\section{Ulcer area}

Ulcer areas were recorded weekly with a digital camera (Sony DSC-H5, 12 megapixels). A standard rule was positioned on the surface of the skin around the ulcers to standardize the captured images and for data analysis. The photographs were analyzed using UTHSCSA ImageTool 3.0 software (developed in the Department of Dental Diagnostic Science at The University of Texas Health Science Center, San Antonio, Texas). A blinded investigator manually delimitated the ulcer areas to calculate the area. The total area was expressed in square centimeters.

In addition, all patients were asked to record their subjective feelings, such as pain, burning and secretions. The level of experienced pain was assessed as follows: pain decreased or remained the same and/or increased. The exudate amount was classified as absent, small, moderate, or large.

\section{Sample collection}

Peripheral blood samples and a biopsy tissue specimen were obtained at the initiation of treat- ment and after 8 weeks (in cases where the ulcer had not healed). After the administration of topical anesthetic (lidocaine spray, 10\%), a biopsy tissue specimen was obtained from the border of the lesion using a disposable, 4-mm-punch instrument. The biopsy tissue specimens were transported in RPMI solution (Gibco by Life Technologies, NY, USA) and processed within $1 \mathrm{~h}$ after sampling. Four milliliters of whole blood was collected from the antecubital vein of each subject and centrifuged (Heraeus Megafuge 40R, Thermo Fisher Scientific Inc., MA) at $2500 \mathrm{rpm}$ for $10 \mathrm{~min}$ to isolate serum, which was frozen at $-70^{\circ} \mathrm{C}$ until cytokine profile analysis.

\section{Cytokine measurement}

Human serum TNF, IL-10, IL-6, IL-1 $\beta$ and IL- 8 levels were quantified using bead-based assays following the same principle as a sandwich immunoassay (BD Cytometric Bead Array; BD Biosciences, San Jose, CA, USA) in accordance with the manufacturer's instructions. Fluorescence from the beads was detected using a BD Accuri C6 flow cytometer system (Becton Dickinson) and analyzed with FCAP Array V3.0 software.

\section{Flow cytometry analysis}

The biopsy tissues were each cut into two pieces, which were each immediately placed into a disposable Medicon disaggregator with a $50-\mu \mathrm{m}$ separator mesh (Becton Dickinson) containing $1 \mathrm{ml}$ of ice-cold PBS and then processed for 50 s using the Medimachine System (Becton Dickinson). Each Medicon unit contained a fixed stainless steel screen with approximately 100 hexagonal holes surrounded by six microblades. The tissue was brought to each hole by a metal rotor inside the Medicon chamber and disaggregated by being passed over the sharpened holes and through the metal screen, while a micropump under the screen supplied liquid and flushed out the holes. The cell suspension was recovered from the Medicon unit using a 5-ml disposable syringe, subsequently filtered through 50- $\mu \mathrm{m}$ Filcon (Becton Dickinson) and $25-\mu \mathrm{m}$ nylon meshes, and placed on ice.

The cell suspensions were stained with fluorescein isothiocyanate (FitC)-conjugated mAb specific for $\mathrm{CD} 3$, phycoerythrin (PE)-conjugated mAb specific for CD8, peridinin chlorophyll (PerCP)-conjugated mAb specific for CD45, allophycocyanin (APC)-conjugated mAb specific for CD4, PE-conjugated mAb specific for CD68 or Alexa Flour 488-conjugated mAb specific for CD31 (BD, Biosciences, San Diego, CA, USA). Briefly, $1 \times 10^{6}$ cells were stained with a fluorochrome-conjugated mAb specific for a cell surface antigen marker for $20 \mathrm{~min}$ in the dark at $4^{\circ} \mathrm{C}$. After the initial staining, 
the cells were washed twice with phosphate-buffered saline (PBS), $\mathrm{pH}$ 7.2. The negative control samples were incubated with isotype-matched antibodies. After incubation, the cells were resuspended in $200 \mu \mathrm{l}$ of PBS for subsequent flow cytometric analysis using an Accuri C6 flow cytometer (BD Biosciences, San Jose, CA, USA) and then analyzed using Flowlo software V10 (Tree Star, San Carlos, CA, USA).

\section{Statistical analysis}

The distribution of the data was tested for normality using the Kolmogorov-Smirnov test. The paired $t$ test and Wilcoxon test were used to compare before and after treatments. $P$-values $<0.05$ were considered significant. Statistical analyses were performed using GraphPad Prism version 5.0 (GraphPad Software, La Jolla, CA, USA).

\section{Results}

\section{A carbohydrate polymer with zinc oxide improves wound healing of CVUs}

A total of 40 patients with a CVU were enrolled in this study. Five patients were excluded because they did not complete the treatment. The mean age of the patients in the study group was estimated to be $59.5 \pm 17.17$ years, while the mean age of the patients in the control group was $62.7 \pm 21.97$ years. The ages of all of the patients ranged from 22 to 89 years, with an average of 61.15 years. Females in the study group were more commonly affected than males $(70.0 \%$ in the study group vs. $45.0 \%$ in the control group). Table I shows the baseline characteristics of the studied patients. At the end of the treatment period ( 8 weeks), complete healing was evident in $5(26.3 \%)$ patients of the study group and in $2(12.5 \%)$ patients of the control group, but this difference was not significant $(p=0.2796)$. A significantly greater reduction in the mean percentage ulcer area from baseline to 8 weeks was associated with the incorporation of a carbohydrate polymer with zinc oxide (Figure 1). Thirteen (68.4\%), 1 (5.3\%) and $0(0 \%)$ patients in the study group and $11(68.7 \%), 0(0 \%)$ and

Table I. Baseline characteristics of the studied patients

\begin{tabular}{|lcc|}
\hline Characteristic & Study group & Control group \\
\hline Age [years] & $59.5 \pm 17.17$ & $62.7 \pm 21.97$ \\
\hline Size of ulcer $\left[\mathrm{cm}^{2}\right]$ & $18.04 \pm 13.36$ & $19.74 \pm 14.07$ \\
\hline $\begin{array}{l}\text { Gender: } \\
\text { Male }\end{array}$ & $6(30.0 \%)$ & $11(55.0 \%)$ \\
\hline Female & $14(70.0 \%)$ & $9(45.0 \%)$ \\
\hline
\end{tabular}

$3(18.8 \%)$ patients in the control group respectively showed partial healing, no improvement or worsening of their ulcers. Furthermore, 19 (100\%) patients in the study group experienced a decrease in pain and burning, while only 6 (37.5\%) patients in the control group reported improvement of those symptoms. Table II shows the ulcer status, level of pain and exudate of all patients in both groups by the end of the treatment period.

\section{Measurement of inflammatory cytokine levels in the serum of patients with a CVU before and after treatment with a carbohydrate polymer with zinc oxide}

To evaluate the systemic inflammatory state of the patients, the concentrations of inflammatory cytokines were measured before and after 8-week treatment with a carbohydrate polymer with zinc oxide plus conventional venous compression. The results showed that patients treated with a carbohydrate polymer with zinc oxide had lower levels of IL-6 ( $p=0.030)$ and IL-8 ( $p=0.002)$ after 8-week treatment with a carbohydrate polymer with zinc oxide compared with the basal samples, whereas patients treated with venous compression alone showed no differences. No differences were found in the levels of TNF, IL-10 or IL-1 $\beta$ in both groups before and after treatment (Figure 2).

\section{Inflammatory cells present in biopsies of patients with a CVU and treated with a carbohydrate polymer with zinc oxide}

After 8 weeks of treatment, the cellular populations present in the biopsies were analyzed using flow cytometry to study the levels of important cells linked to the inflammatory state. Likewise, we used the leukocyte common antigen $C D 45^{+}$to study immune cell infiltration. Using this marker, we found that patients treated with a carbohydrate polymer with zinc oxide had a reduced percentage of $\mathrm{CD} 45^{+}$cells $(p=0.003)$ compared with

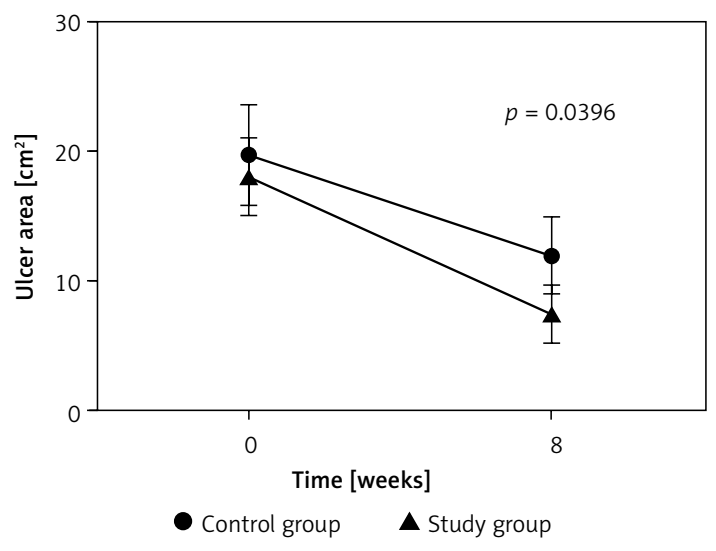

Figure 1. Rates of reduction in the mean surface areas of the ulcers of both groups 
M.A. Moreno-Eutimio, L. Espinosa-Monroy, T. Orozco-Amaro, Y. Torres-Ramos, A. Montoya-Estrada, J.J. Hicks, E. Rodríguez-Ayala, P. Del Moral, J. Moreno, J. Cueto-García

Table II. Ulcer status at the end of the treatment period

\begin{tabular}{|c|c|c|c|}
\hline Variable & $\begin{array}{l}\text { Study group } \\
\quad(n=19)\end{array}$ & $\begin{array}{l}\text { Control group } \\
\quad(n=16)\end{array}$ & $P$-value* \\
\hline \multicolumn{4}{|l|}{ Ulcer status: } \\
\hline Complete healing & $5(26.3 \%)$ & $2(12.5 \%)$ & 0.4150 \\
\hline Partial healing & $13(68.4 \%)$ & 11 (68.7\%) & 1.0000 \\
\hline No improvement & $1(5.3 \%)$ & $0(0 \%)$ & 1.0000 \\
\hline Worsening & $0(0 \%)$ & $3(18.8 \%)$ & 0.0856 \\
\hline \multicolumn{4}{|l|}{ Level of pain: } \\
\hline Pain decreased & 19 (100.0\%) & $6(37.5 \%)$ & $<0.0001$ \\
\hline Pain remained the same & $0(0 \%)$ & $7(43.7 \%)$ & 0.0017 \\
\hline Pain increased & $0(0 \%)$ & $3(18.8 \%)$ & 0.0856 \\
\hline \multicolumn{4}{|l|}{ Exudate: } \\
\hline Absent & $19(100.0 \%)$ & $13(81.3 \%)$ & 0.0856 \\
\hline Small & $0(0 \%)$ & $2(12.5 \%)$ & 0.2017 \\
\hline Moderate & $0(0 \%)$ & $1(6.2 \%)$ & 0.4571 \\
\hline
\end{tabular}

${ }^{\star}$ Fisher's exact test.

basal values. The control group showed no differences (Figure 3).

The percentages of $\mathrm{T}$ helper lymphocyte populations $\left(\mathrm{CD}^{2} 5^{+}, \mathrm{CD}^{+}\right.$and $\left.\mathrm{CD} 4^{+}\right)$were not different before and after treatment in both groups (Figure 3).

Interestingly, cytotoxic $\mathrm{T}$ cells $\left(\mathrm{CD}_{4} 5^{+}, \mathrm{CD}^{+}\right.$ and $\left.\mathrm{CD}^{+}\right)$were increased in patients treated with venous compression treatment alone compared with the basal values (Figure 3).

Analysis of macrophages (CD68+) showed that the percentages of this population were not significantly different before and after treatment in both groups (Figure 3).

The endothelial cell population $\left(\mathrm{CD} 31^{+}\right)$was then investigated, and a significant increase in CD3 $1^{+}$cells ( $\left.p=0.045\right)$ was found in patients treated with a carbohydrate polymer with zinc oxide plus compression after 8 weeks of treatment. No difference was observed before and after treatment in the control group (Figure 3).

\section{Discussion}

This study shows, for the first time, that a carbohydrate polymer with zinc oxide enhances healing and the quality of life of patients with CVUs, due to its anti-inflammatory activity. Our results are supported by data reported in animals models. A beneficial effect of carbohydrate polymer with zinc oxide was observed after 8 weeks of twice-daily, topical application by the patients or their relatives complemented with venous compression.

The clinical results obtained in the present study showed a distinct improvement in quali- ty of life (measured by the level of pain, burning and absence of exudate) in the patients treated with a carbohydrate polymer with zinc oxide, as compared with the control group. This was very important for the patients because they were able to sleep and walk normally and conceivably could partially return to their normal life without the need of additional medications. A carbohydrate polymer with zinc oxide therapy also has the advantage that it can be easily applied by patients or their relatives without the need of specialized personnel, sterile instruments, etc., thus producing important savings for these families.

The decreased levels of pain and increased quality of life of patients with CVU may be related to lower levels of pro-inflammatory cytokines (IL-6 and IL-8) and lower leukocyte infiltrates into the ulcer that were observed in patients treated with a carbohydrate polymer with zinc oxide.

Inflammation is a highly regulated process that provides a quick response to pathogens or host stress signals produced after tissue damage or other types of insults [21, 22]. It has been shown, however, that excessive chronic, protracted inflammation causes further tissue damage, collagenolysis and delayed wound repair in non-healing chronic lesions such as CVUs [9].

Although the etiopathogenesis of CVUs and the relationship between cytokine levels before and after compression therapy of CVUs or in healthy tissues is not well understood, it is well known that some cytokines and growth factors are key regulators of the normal wound healing process [14, 23-25]. Elevated inflammatory cytokine lev- 
A

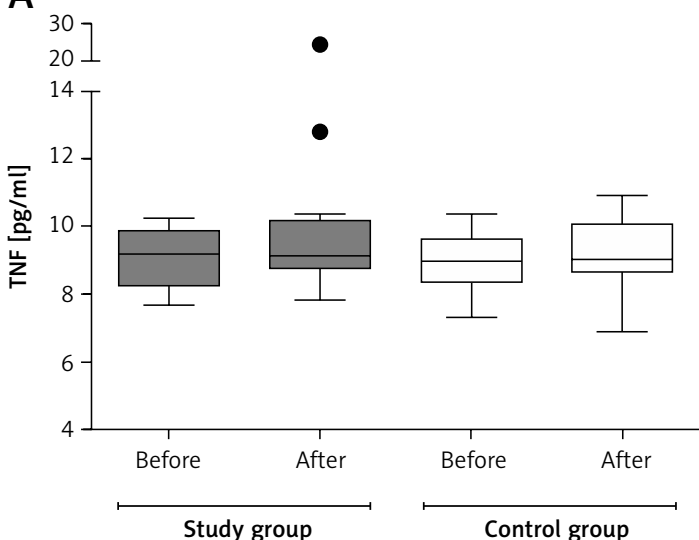

C

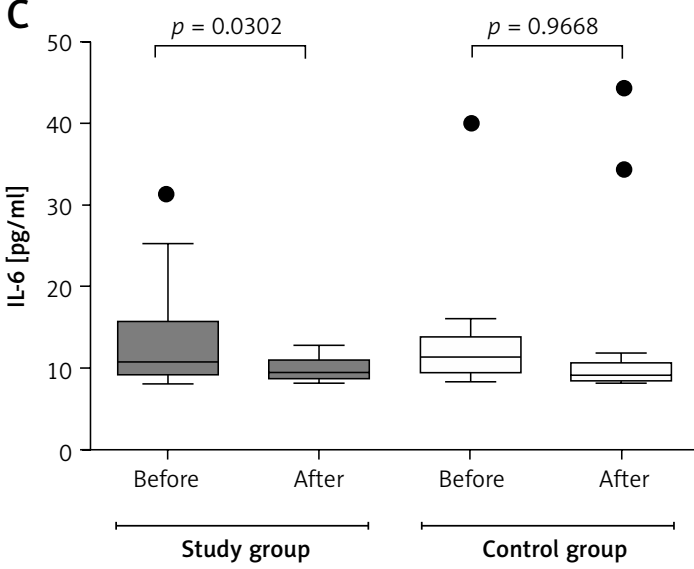

E

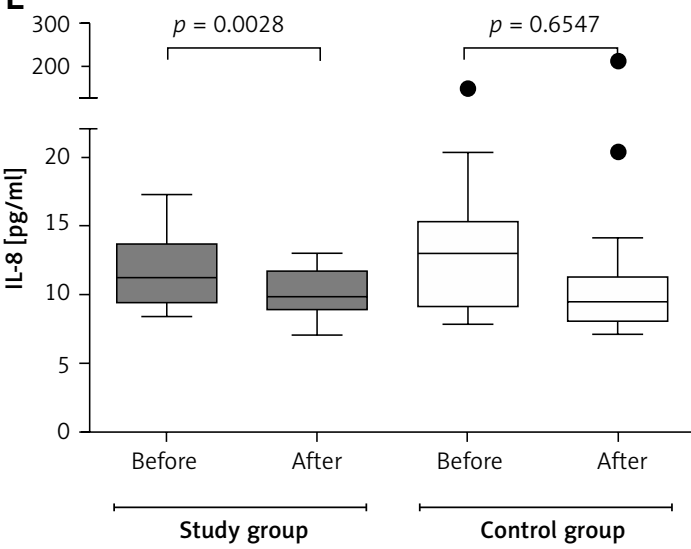

els have been implicated in the pathogenesis of non-healing CVUs [9]. In this regard, our data show that patients treated with carbohydrate polymer with zinc oxide therapy had lower levels of systemic IL-8, which is an important marker of inflammation related to neutrophil activation and of the activation of innate immune reactions related to the lack of wound healing due to an altered, chronically inflamed environment $[24,26]$.

We found lower levels of IL-6 in the sera of the study group compared with those of the control group. IL-6 is an important cytokine in the inflammatory process because it is a crucial regulator
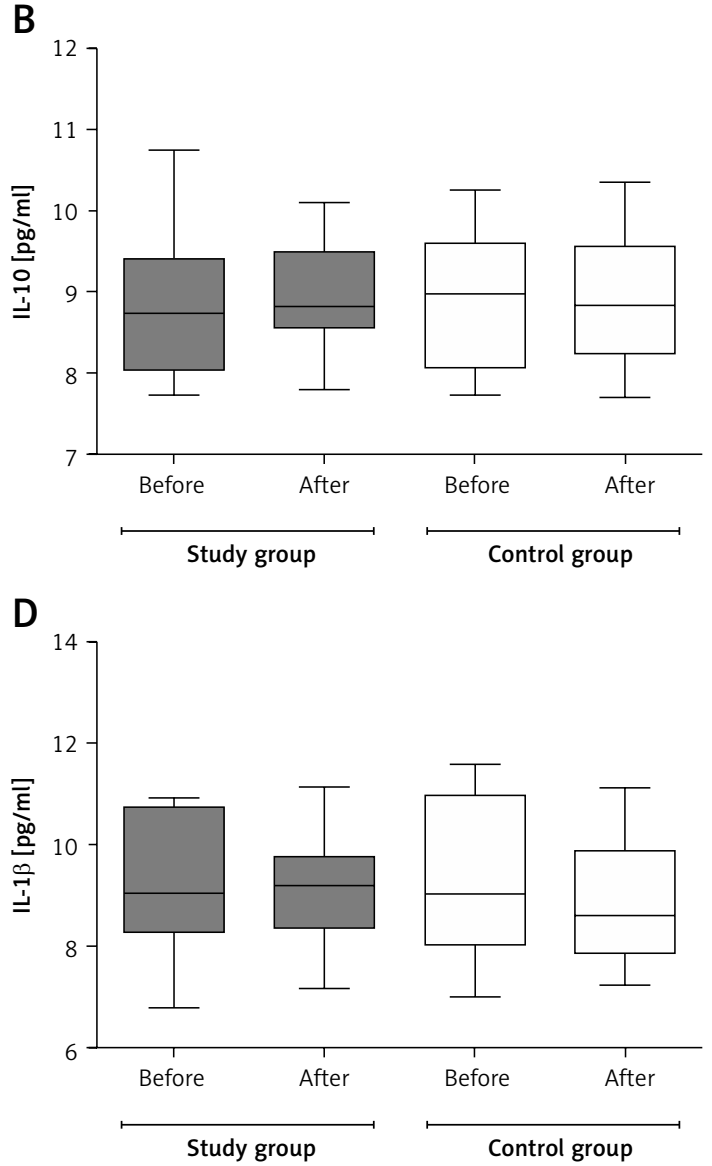

Figure 2. Levels of inflammatory cytokines in the sera of patients with CVUs and treated with a carbohydrate polymer with zinc oxide. The levels of cytokines in the sera of patients treated with compression (control group, $n=16$ ) or a carbohydrate polymer with zinc oxide with venous compression (study group, $n=19$ ) for 8 weeks were measured using a BD Human Inflammation CBA Kit. The data were analyzed using Student's $t$-test with GraphPad Prism software (GraphPad Software, Inc.). Differences with a $p$-value equal to or below 0.05 were considered statistically significant

that may produce different effects; sometimes it acts as a defense mechanism, but in chronic inflammation, its role is altogether different [27, 28].

The study of the cellular components of the ulcerated tissues of CVU patients treated with both therapies revealed important differences in the percentages of total $\mathrm{CD} 45^{+}$cell populations. Thus, there was a reduced presence of immune cells (CD45+ cells) in the wounds of patients after treatment with a carbohydrate polymer with zinc oxide, whereas there were no such differences in the control group (without carbohydrate polymer with zinc oxide therapy treatment). These data 
A

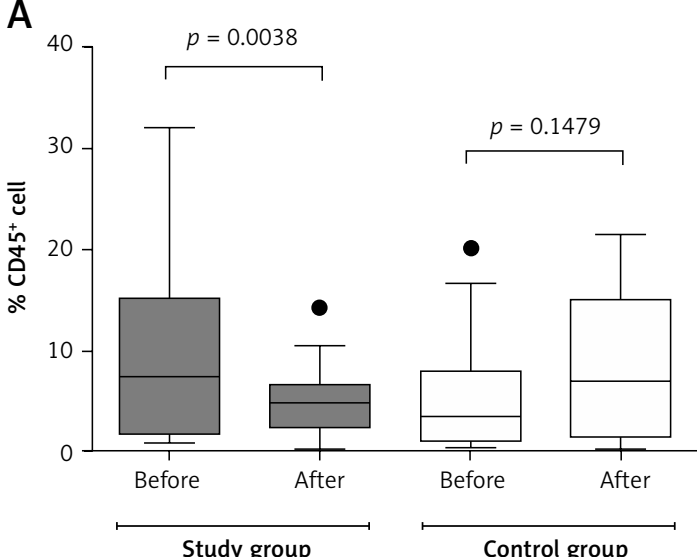

C

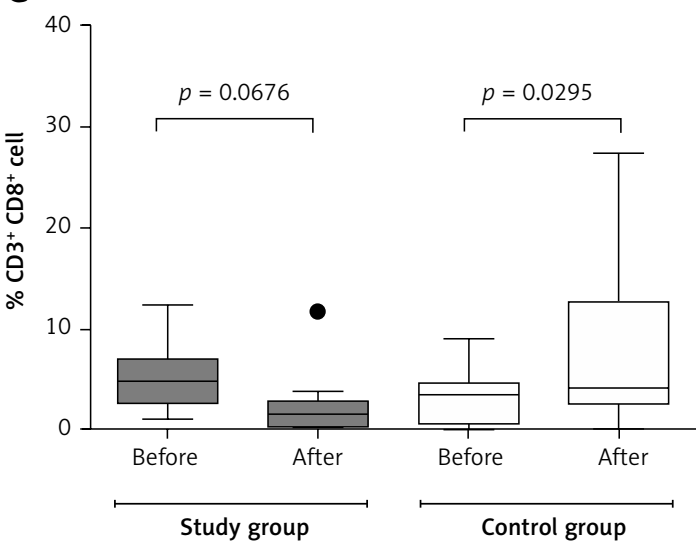

E

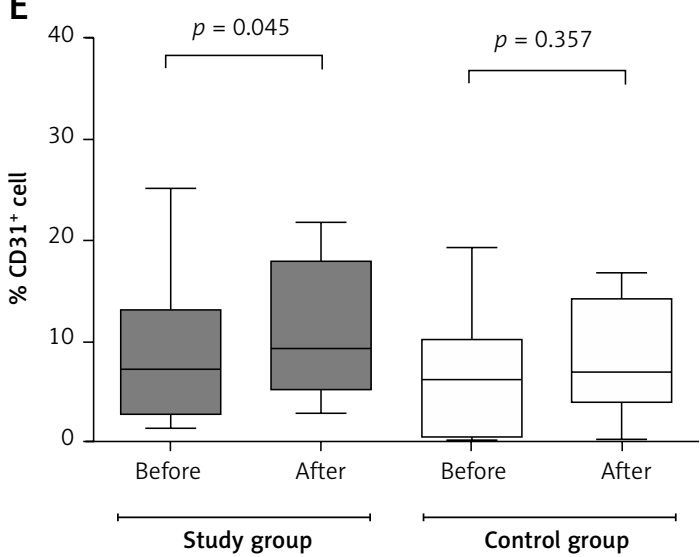

B

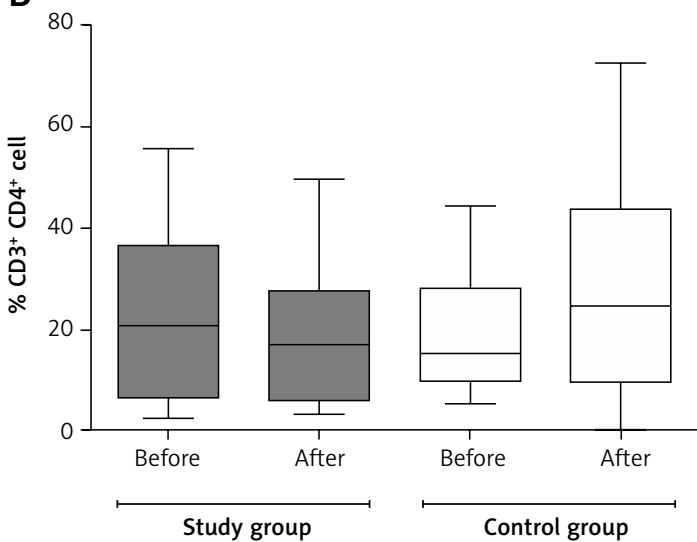

D

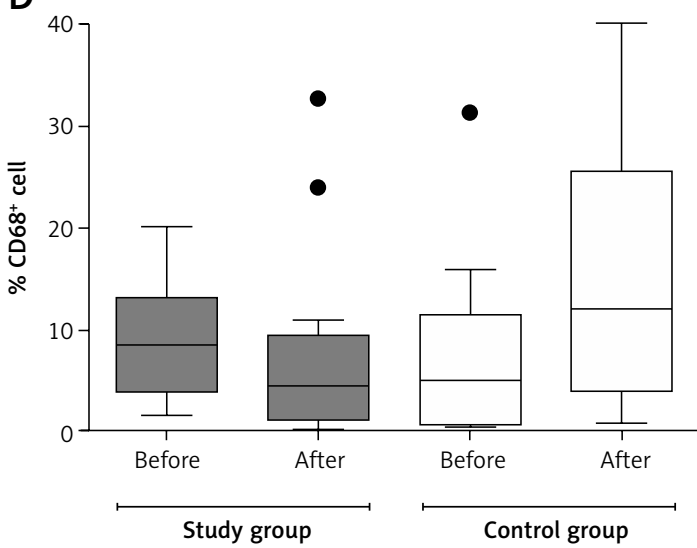

Figure 3. Analysis of cell subpopulations present in biopsies from patients with CVUs after both treatments. We analyzed the different cell subpopulations in the biopsy tissue specimens using flow cytometry. The number of total leukocytes $\left(\mathrm{CD} 45^{+}\right)$ (A), $\mathrm{T}$ helper lymphocytes $\left(\mathrm{CD}^{2} 5^{+}, \mathrm{CD}^{+}, \mathrm{CD}^{+}\right)$ (levels shown as the percentage of $\mathrm{CD}^{+}$cells) (B), $\mathrm{T}$ cytotoxic lymphocytes $\left(\mathrm{CD}_{4} 5^{+}, \mathrm{CD}^{+}\right.$and $\left.\mathrm{CD} 8^{+}\right)$ (levels shown as the percentage of $\mathrm{CD} 8^{+}$cells) (C), macrophages (levels shown as the percentage of CD34+ cells) (D) and endothelial cells (levels shown as the percentage of $\mathrm{CD} 31^{+}$cells) (E) from patients treated with compression (control group, $n=13$ ) or carbohydrate polymer with zinc oxide with venous compression (study group, $n=14$ ). The analysis was performed before and after 8 weeks of treatment for each group. The data were analyzed using a non-parametric $t$ test and a Wilcoxon signed-rank test using GraphPad Prism software (GraphPad Software, Inc.). Differences with a $p$-value equal to or below 0.05 were considered statistically significant

centages of $\mathrm{T}$ cytotoxic lymphocyte populations were not different after treatment with a carbohydrate polymer with zinc oxide. The involvement of T lymphocytes in skin wound healing has not been studied extensively. Studies of cytotoxic $T$ lymphocyte depleted mice or rats have generally shown enhanced wound healing, suggesting that cytotoxic $T$ lymphocytes have an inhibitory effect 
on wound healing in terms of collagen deposition $[29,30]$. Wounds in cytotoxic T lymphocyte deficient mice showed significantly decreased infiltration of neutrophils and macrophages along with down-regulated expression of IL-1 $\beta$, IL-6, TNF- $\alpha$, CXCL1 and CCL2 as compared to wild type mice [31]. Therefore cytotoxic T cells increased in patients treated with venous compression treatment alone compared with the basal values. It could be considered to have a poor outcome.

The CD31 molecule was investigated as a marker of angiogenesis. In fact, CD31 represents a highly specific marker for vascular and endothelial cells [32], and it was found that the carbohydrate polymer with zinc oxide-treated group had an increased percentage of these cells. This finding correlates well with the fact that patients treated with a carbohydrate polymer with zinc oxide exhibited improved wound healing; however, it is very important to measure the levels of growth factors known to be related to the re-epithelialization process to determine whether this type of treatment also induces the release of these molecules.

Several studies have demonstrated that some carbohydrate polymers exert anti-inflammatory activity, and it is known that $\mathrm{ZnO}$ has immunoregulatory properties. In addition, a carbohydrate polymer with zinc oxide has antibacterial activity, and $\mathrm{ZnO}$ improves re-epithelialization, both of which are important factors during a successful wound healing process.

Thus, this study shows, for the first time, that a carbohydrate polymer with zinc oxide might be useful for treating chronic wounds with abnormal delayed healing, such as CVUs, and that the antimicrobial activity of the main polysaccharide and $\mathrm{ZnO}$ components of the carbohydrate polymer with zinc oxide formula might be effective in treating infected wounds. In this regard, bacteriological studies of our group that are now in progress have confirmed a definite potent antibacterial activity of a carbohydrate polymer with zinc oxide.

In conclusion, the results described in this study, which are supported by previous experimental results [16], suggest that the use of a carbohydrate polymer with zinc oxide improves healing and quality of life of patients and reduces inflammation in patients with CVUs when used with standard venous compression treatment.

\section{Acknowledgments}

The authors are grateful to all patients who participated in this study.

\section{Conflict of interest}

The authors declare no conflict of interest.

\section{References}

1. Carter MJ. Economic evaluations of guideline-based or strategic interventions for the prevention or treatment of chronic wounds. Appl Health Econ Health Policy 2014; 12: 373-89.

2. Raffetto JD, Marston WA. Venous ulcer: what is new? Plast Reconstr Surg 2011; 127 Suppl 1: 279S-88S.

3. Hopman WM, VanDenKerkhof EG, Carley ME, Kuhnke JL, Harrison MB. Factors associated with health-related quality of life in chronic leg ulceration. Qual Life Res 2014; 23: 1833-40.

4. Gloviczki P, Comerota AJ, Dalsing MC, et al. The care of patients with varicose veins and associated chronic venous diseases: clinical practice guidelines of the Society for Vascular Surgery and the American Venous Forum. J Vasc Surg 2011; 53: 2S-48S.

5. O'Meara S, Cullum N, Nelson EA, Dumville JC. Compression for venous leg ulcers. Cochrane Database Syst Rev 2012; 11: CD000265.

6. Zenilman J, Valle MF, Malas MB, et al. Chronic venous ulcers: a comparative effectiveness review of treatment modalities. AHRQ Comparative Effectiveness Reviews 2013; 127.

7. Eming SA, Krieg T, Davidson JM. Inflammation in wound repair: molecular and cellular mechanisms. J Invest Dermatol 2007; 127: 514-25.

8. DiPietro LA. Wound healing: the role of the macrophage and other immune cells. Shock 1995; 4: 233-40.

9. Raffetto JD. Inflammation in chronic venous ulcers. Phlebology 2013; 28 Suppl 1: 61-7.

10. Mwaura B, Mahendran B, Hynes N, et al. The impact of differential expression of extracellular matrix metalloproteinase inducer, matrix metalloproteinase-2, tissue inhibitor of matrix metalloproteinase- 2 and PDGF-AA on the chronicity of venous leg ulcers. Eur J Vasc Endovasc Surg 2006; 31: 306-10.

11. Raffetto JD. Dermal pathology, cellular biology, and inflammation in chronic venous disease. Thromb Res 2009; 123 Suppl 4: S66-71.

12. Ashcroft GS, Jeong MJ, Ashworth JJ, et al. Tumor necrosis factor-alpha (TNF-alpha) is a therapeutic target for impaired cutaneous wound healing. Wound Repair Regen 2012; 20: 38-49.

13. Streit M, Beleznay Z, Braathen LR. Topical application of the tumour necrosis factor-alpha antibody infliximab improves healing of chronic wounds. Int Wound J 2006; 3: 171-9.

14. Wallace HJ, Stacey MC. Levels of tumor necrosis factor-alpha (TNF-alpha) and soluble TNF receptors in chronic venous leg ulcers: correlations to healing status. J Invest Dermatol 1998; 110: 292-6.

15. Loots MA, Lamme EN, Zeegelaar J, Mekkes JR, Bos JD, Middelkoop E. Differences in cellular infiltrate and extracellular matrix of chronic diabetic and venous ulcers versus acute wounds. J Invest Dermatol 1998; 111: 850-7.

16. Moreno-Eutimio MA, Nieto-Velázquez NG, EspinosaMonroy L, et al. Potent anti-inflammatory activity of carbohydrate polymer with oxide of zinc. Biomed Res Int 2014; 2014: 712312.

17. Cueto J, Barrientos T, Rodriguez E, Del Moral P. A new biodegradable adhesive for protection of intestinal anastomoses. Preliminary communication. Arch Med Res 2011; 42: 475-81.

18. Cueto J, Barrientos T, Rodriguez E, et al. Further experimental studies on a biodegradable adhesive for protection of colorectal anastomosis. Arch Med Res 2014; 45: 331-6. 
19. Jones N, Ray B, Ranjit KT, Manna AC. Antibacterial activity of ZnO nanoparticle suspensions on a broad spectrum of microorganisms. FEMS Microbiol Lett 2008; 279: 71-6.

20. Sargeant HR, McDowall KJ, Miller HM, Shaw MA. Dietary zinc oxide affects the expression of genes associated with inflammation: transcriptome analysis in piglets challenged with ETEC K88. Vet Immunol Immunopathol 2010; 137: 120-9.

21. Medzhitov R. Inflammation 2010: new adventures of an old flame. Cell 2010; 140: 771-6.

22. Plytycz B, Seljelid R. From inflammation to sickness: historical perspective. Arch Immunol Ther Exp (Warsz) 2003; 51: 105-9.

23. Claudy AL, Mirshahi M, Soria C, Soria J. Detection of undegraded fibrin and tumor necrosis factor-alpha in venous leg ulcers. J Am Acad Dermatol 1991; 25: 623-7.

24. Peral MC, Rachid MM, Gobbato NM, Huaman Martinez MA, Valdez JC. Interleukin-8 production by polymorphonuclear leukocytes from patients with chronic infected leg ulcers treated with Lactobacillus plantarum. Clin Microbiol Infect 2010; 16: 281-6.

25. Pukstad BS, Ryan L, Flo TH, et al. Non-healing is associated with persistent stimulation of the innate immune response in chronic venous leg ulcers. J Dermatol Sci 2010; 59: 115-22.

26. Engelhardt E, Toksoy A, Goebeler M, Debus S, Brocker EB, Gillitzer R. Chemokines IL-8, GROalpha, MCP-1, IP-10, and Mig are sequentially and differentially expressed during phase-specific infiltration of leukocyte subsets in human wound healing. Am J Pathol 1998; 153: $1849-60$.

27. Ambrosch A, Lobmann R, Pott A, Preissler J. Interleukin-6 concentrations in wound fluids rather than serological markers are useful in assessing bacterial triggers of ulcer inflammation. Int Wound J 2008; 5: 99-106.

28. Gabay C. Interleukin-6 and chronic inflammation. Arthritis Res Ther 2006; 8 Suppl 2: S3.

29. Davis PA, Corless DJ, Aspinall R, Wastell C. Effect of CD4(+) and CD8(+) cell depletion on wound healing. $\mathrm{Br}$ J Surg 2001; 88: 298-304.

30. Efron JE, Frankel HL, Lazarou SA, Wasserkrug HL, Barbul $A$. Wound healing and T-lymphocytes. J Surg Res 1990; 48: 460-3.

31. Chen L, Mehta ND, Zhao Y, DiPietro LA. Absence of CD4 or CD8 lymphocytes changes infiltration of inflammatory cells and profiles of cytokine expression in skin wounds, but does not impair healing. Exp Dermatol 2014; 23: 189-94.

32. DeLisser HM, Christofidou-Solomidou M, Strieter RM, et al. Involvement of endothelial PECAM-1/CD31 in angiogenesis. Am J Pathol 1997; 151: 671-7. 